\title{
GUÍAS DE PRÁCTICA CLÍNICA DE LA SERV: TRATAMIENTO DE LA DEGENERACIÓN MACULAR ASOCIADA A LA EDAD (DMAE) EXUDATIVA
}

\author{
GUIDELINES OF CLINICAL PRACTICE OF THE SERV: \\ TREATMENT OF EXUDATIVE AGE-RELATED MACULAR \\ DEGENERATION (AMD)
}

\author{
RUIZ-MORENO JM ${ }^{1}$, ARIAS-BARQUET L ${ }^{2}$, ARMADÁ-MARESCA F ${ }^{3}$, BOIXADERA-ESPAX A ${ }^{4}$, \\ GARCÍA-LAYANA A ${ }^{5}$, GÓMEZ-ULLA-DE-IRAZAZÁBAL F ${ }^{6}$, MONÉS-CARILLA J ${ }^{7}$, PIÑERO- \\ BUSTAMANTE $\mathrm{A}^{8}$, SUÁREZ-DE-FIGUEROA $\mathrm{M}^{9}$
}

\begin{abstract}
RESUMEN
Objetivo: La Degeneración Macular Asociada a la Edad (DMAE) en su forma húmeda supone una grave enfermedad que condiciona ceguera legal en muchos pacientes y con mal pronóstico si no es tratada. Pretendemos establecer una guía de actuación clínica con las diferentes opciones terapeúticas que existen en el momento actual, que puedan ayudar al oftalmólogo en su práctica clínica.

Métodos: Un grupo de expertos en retina médica seleccionados por la SERV han evaluado los resultados de los diferentes estudios publicados con los fármacos actualmente disponibles, llegando a un
\end{abstract}

\begin{abstract}
Objective: Age related macular degeneration (ARMD) in its neovascular form is a serious disease which produces legal blindness in many patients with poor prognosis if left untreated. We intend to establish a clinical guide with the different therapeutic options that exist nowadays, which may help the ophthalmologists in their clinical practice.

Methods: A group of medical retina experts selected by SERV have evaluated the results of different published studies with the drugs currently available, obtaining an evidence-based consensus. Some recommendations have been established for diagno-
\end{abstract}

Recibido: 1/5/09. Aceptado: 30/6/09

1 Catedrático de Oftalmología de la Universidad de Castilla - La Mancha. Instituto Oftalmológico Alicante (VISSUM). Red Temática de Investigación Cooperativa (RTICS) en Oftalmología. Albacete. España.

2 Profesor Asociado de la Universitat de Barcelona. Hospital Universitari de Bellvitge. Institut de la Màcula i de la Retina. Centro Médico Teknon. Barcelona. España.

3 Jefe de Sección del Servicio de Oftalmología. Hospital La Paz (Madrid). Coordinador del Servicio de Oftalmología. Sanatorio San Francisco de Asís. Madrid. España.

4 Hospilar Universitari Vall d'Hebron (Barcelona). Instituto de Cirugía Ocular Avanzada de Barcelona (ICOAB). Barcelona. España.

5 Profesor Titular de Oftalmología. Clínica Universitaria y Universidad de Navarra. Red Temática de Investigación Cooperativa (RTICS) en Oftalmología. Pamplona. España.

6 Catedrático de Oftalmología de la Universidad de Santiago de Compostela. Hospital Provincial de Conxo (Santiago de Compostela). Instituto Tecnológico de Oftalmología (ITO). Presidente de la Sociedad Española de Retina yVítreo (SERV). Santiago de Compostela. España.

7 Institut de la Màcula i de la Retina. Centro Médico Teknon. Barcelona. España.

8 Catedrático de Oftalmología de la Universidad de Sevilla. Jefe de Servicio de Oftalmología del Hospital Universitario de Valme (Sevilla). Expresidente de la Sociedad Española de Retina y Vítreo. Sevilla. España.

9 Directora del Departamento de Retina y Vítreo de VISSUM Madrid. Adjunto del Departamento de Retina y Vítreo del Hospital Ramón y Cajal (Madrid). Profesora Asociada de la Universidad de Alcalá de Henares. Madrid. España.

Correspondencia:

José María Ruiz Moreno

Facultad de Medicina

Avenida de Almansa, 14

02006 Albacete

España

E-mail: JoseMaria.Ruiz@uclm.es 
consenso basado en la evidencia. Se han establecido unas recomendaciones para el diagnóstico, tratamiento y seguimiento de los enfermos con DMAE húmeda.

Resultados: La inyección intravítrea de ranibizumab a la dosis de $0,5 \mathrm{mg}$ permite obtener mejorías significativas de la agudeza visual en lesiones subfoveales, según los datos obtenidos de estudios con máximo nivel de evidencia. Debe ser considerado como el fármaco de primera elección. El empleo de bevacizumab, fármaco sin indicación aprobada para uso intraocular, ni para el tratamiento de la DMAE húmeda, puede aportar un beneficio más próximo a los resultados obtenidos con el ranibizumab que a los obtenidos con la terapia fotodinámica (TFD) y el pegaptanib. El uso de pegaptanib sódico en inyección intravítrea a la dosis de $0,3 \mathrm{mg}$ como tratamiento de lesiones subfoveales permite obtener resultados parecidos a la TFD, pero en un abanico más amplio de lesiones

Conclusiones: Los resultados de los estudios basados en la evidencia constituyen una buena guía de actuación en el tratamiento de esta enfermedad.

Palabras clave: Degeneración macular asociada a la edad (DMAE), ranibizumab, bevacizumab, pegaptanib sódico, terapia fotodinámica, verteporfin, VEGF. sis, treatment and monitoring of patients with neovascular ARMD.

Results: The intravitreal injection of ranibizumab at a dosage of $0.5 \mathrm{mg}$ produces significant improvement of visual acuity in subfoveal lesions, according to data obtained from studies with the highest level of evidence. It should be considered as a first choice drug. The use of bevacizumab, a drug with not approved indication for intraocular use, nor for the treatment of neovascular ARMD can reach a result which is closer to the ones obtained with ranibizumab than to those obtained with photodynamic therapy (PDT) and pegaptanib. The use of intravitreal pegaptanib sodium in intravitreal injection at a dosage of $0.3 \mathrm{mg}$ as a treatment for subfoveal lesions can obtain similar results to the TFD, but with a wider range of injuries.

Conclusion: The results of the evidence-based studies are a good guide for the treatment of this disease (Arch Soc Esp Oftalmol 2009; 84: 333-344).

Key words: Age-related Macular Degeneration (AMD), ranibizumab, bevacizumab, pegaptanib, photodynamic therapy, verteporfin, VEGF.

\section{DESARROLLO DE LA GUÍA}

\section{Definición de objetivos}

El objetivo de la Guía de tratamiento de la DMAE húmeda es ofrecer una herramienta de referencia a los oftalmólogos/retinólogos para facilitarles el manejo de los pacientes afectos de esta enfermedad en su práctica clínica diaria, basada en los conocimientos científicos actuales.

Tanto por parte de los profesionales como de los autores, existe un objetivo final de mejorar la calidad de vida de nuestros pacientes con esta enfermedad a través de una mejora en el diagnóstico, el tratamiento y la rehabilitación, que permita obtener la mejor agudeza visual posible.

Los autores han ofrecido un consenso en relación a las perspectivas actuales respecto de los beneficios y riesgos de las estrategias de tratamiento que existen en la actualidad.

\section{Escenarios clínicos a los que se refiere la Guía}

La enfermedad objeto de esta guía es la Degeneración Macular Asociada a la Edad (DMAE) tipo exudativa. La población de aplicación son pacientes con 50 o más años de edad.

\section{Métodos}

Se ha realizado una búsqueda de las publicaciones científicas en relación con el objeto de esta Guía, de forma que las recomendaciones terapéuticas estén basadas en conocimientos científicos.

La información científica empleada ha sido de diferentes niveles de evidencia. La evidencia más alta es la basada y proporcionada por ensayos clínicos controlados, aleatorizados, multicéntricos, o bien por un estudio de metaanálisis de ensayos clínicos con estas características. El segundo nivel 
estaría constituido por ensayos clínicos controlados sin aleatorización. En este grupo también podemos incluir las publicaciones de análisis de casos y controles con un diseño adecuado. El nivel de evidencia menor lo ofrecen los estudios descriptivos, presentación de casos clínicos y las opiniones de expertos plasmadas en la literatura científica.

\section{Métodos de consenso utilizados para la formulación de recomendaciones}

La reunión del Grupo de trabajo permitió obtener un consenso por parte de todos los participantes en las recomendaciones realizadas y contenidas en esta Guía.

\section{Validación de la Guía}

Esta Guía ha sido validada por la Sociedad Española de Retina y Vítreo.

\section{Validación por agentes externos}

La validación de esta guía ha sido realizada por los siguientes revisores:
- Alfaro, Virgil (Estados Unidos).

- Saravia, Mario (Argentina).

- Corcóstegui Guraya, Borja (España).

- Piñero Bustamante, Antonio (España).

\section{ESQUEMA DE MANEJO DE LA DMAE}

\section{DMAE atrófica}

\section{Sintomatología}

- Pérdida progresiva de agudeza visual.

- Dificultad para la lectura.

- Metamorfopsia.

\section{Clínica}

Hallazgos en el fondo de ojo:

- Drusas duras: lesiones retinianas profundas de color amarillento y bien delimitadas. Representan la fase más precoz de la DMAE atrófica.

\section{ALGORITMO 1}

\section{Clasificación general}

MAE (maculopatía asociada a la edad)

Signos clínicos:

" Drusas duras

"Drusas blandas

- Drusas calcificadas

" Dispersión de pigmento

\section{DMAE atrófica}

Signos clínicos:

- Drusas

- Dispersión pigmento

" Atrofia del EPR

\section{DMAE exudativa}

\section{Signos clínicos:}

"Drusas

- Hemorragia subretiniana o intraretiniana

- Desprendimiento de retina exudativo

- Fibrosis subretiniana

= Desprendimiento del EPR

Algoritmos de manejo de la DMAE. 
- Drusas blandas: lesiones retinianas de color amarillento con bordes menos definidos que las drusas duras y mayores en tamaño que éstas.

- Desprendimiento drusenoide del EPR: no es más que la coalescencia de drusas blandas, que en ocasiones simula la forma de un desprendimiento del EPR.

- Acúmulos de pigmento: indican más severidad de las lesiones y se observan con frecuencia adyacentes a las drusas blandas.

- Drusas calcificadas: drusas blandas en fase de reabsorción por degeneración parcial de las células del EPR localizadas sobre ellas.

- Atrofia del EPR: fase final de esta forma de la enfermedad, a la que se llega por degeneración de las células de EPR sobre las drusas.

\section{Pruebas especiales}

El diagnóstico de DMAE atrófica se realiza en base a la exploración biomicroscópica de la retina. La identificación de cualquiera de los signos descritos en el apartado anterior es suficiente para establecer el diagnóstico.

Sólo en aquellos casos con desprendimientos drusenoides del ERP, es aconsejable descartar la presencia de una membrana neovascular coroidea. A tal efecto, serían útiles tanto la angiografía con fluoresceína (AGF) como el estudio con tomografía de coherencia óptica (OCT). Incluso, algunos autores aconsejan realizar una angiografía con verde de indocianina en estos casos, para descartar una membrana neovascular coroidea oculta.

\section{Diagnóstico diferencial}

Debe establecerse con enfermedades que cursen con drusas o con aquellas que producen atrofias del complejo EPRcoriocapilar. Entre ellas, cabe destacar distrofias como las drusas autosómicas dominantes o la atrofia areolar central.

\section{Tratamiento}

En las fases más precoces de la enfermedad, no es necesario hacer ningún tratamiento específico, aunque se aconseja una dieta rica en zeaxantina y luteína, presentes en general en frutas y verduras, pero a mayor concentración en las espinacas, el brécol, los berros, el maíz, la naranja y la yema de huevo. Los suplementos de zeaxantina y luteína parecen aumentar la concentración de éstos pigmentos en la mácula, haciéndola más resistente a los radicales libres. Otra de las recomendaciones dietéticas son el pescado azul y los frutos secos (nueces), debido a su alto contenido en ácidos grasos poliinsaturados de cadena larga de la familia del omega-3. De ellos, es el ácido docosahexaenoico (DHA) el que parece más directamente implicado en la prevención de las lesiones retinianas inducidas por la edad.

Cuando el estudio de fondo revela la presencia de drusas blandas grandes $(\geq 125$ micras $=$ grosor de una vena en el borde del nervio óptico) y alteraciones pigmentarias, el riesgo de progresión hacia fases avanzadas de la DMAE es alto. Por ello, se aconseja tratamiento con vitaminas antioxidantes (vitamina $\mathrm{E}, \mathrm{C}$ y betacarotenos) y suplementos

\section{ALGORITMO 2}

Pautas de tratamiento DMAE atrófica

\section{Dusas blandas bilaterales mayores de 125 micras con o sin alteración pigmentaria}

\section{Drusas en ojo contralateral \\ a DMAE exudativa o atrofia severa del EPR}

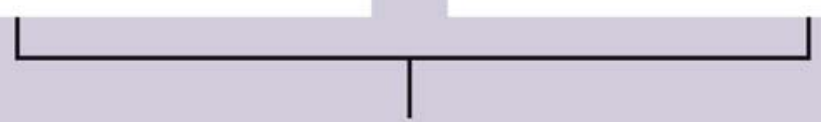

\section{Antioxidantes}

Algoritmos de manejo de la DMAE. 
minerales (zinc). No obstante, ninguno de los fármacos aprobados en nuestro país contiene dosis tan altas como la utilizadas en los ensayos clínicos en los que se ha demostrado que, si bien no previenen la enfermedad, estas sustancias pueden disminuir la velocidad de la progresión hacia las formas avanzadas y la pérdida visual (AREDS). Tal vez las respuestas a los suplementos del estudio AREDS no sean uniformes en todos los pacientes y guarden relación con el genotipo $\mathrm{CFH}$ (factor $\mathrm{H}$ del complemento).

Estos suplementos vitamínicos también están indicados en pacientes que ya presentan una DMAE severa en un ojo, tanto exudativa como atrófica, independientemente del tamaño de las drusas del ojo menos afecto, porque el riesgo de progresión es alto.

Es importante destacar que las dosis altas de vitaminas antioxidantes y minerales no están exentas de riesgo. Por ejemplo, no deben administrase complejos vitamínicos con betacarotenos (provitamina A) en pacientes fumadores o exfumadores de menos de 8 años de cese del consumo, ya que aumenta el riesgo de cáncer de pulmón. La vitamina $\mathrm{E}$ aumenta el riego de fallo cardiaco en pacientes con enfermedades vasculares, y el zinc puede producir enteropatía.

En el momento actual no existe ningún tratamiento que haya demostrado una eficacia clínica importante con capacidad de mejoría o de detener la progresión de la enfermedad en esta modalidad de DMAE.

\section{DMAE exudativa}

\section{Sintomatología}

- Pérdida brusca y progresiva de agudeza visual.

- Escotoma central.

- Dificultad para la lectura.

- Metamorfopsia.

- Fotopsias.

\section{Clínica}

Hallazgos en el área macular:

- Desprendimiento de retina exudativo.

- Desprendimiento seroso del epitelio pigmentario de la retina.

- Hemorragia intrarretiniana.
- Hemorragia subretiniana.

- Hemorragia sub-EPR.

- Exudados lipídicos.

Clasificación de las membranas neovasculares coroideas según localización:

- Subfoveales. Afectan al centro de la fóvea.

- Yuxtafoveales. Entre 1 y 200 micras del centro de la fóvea.

- Extrafoveales. A más de 200 micras del centro de la fóvea.

- Yuxtapapilares. Adyacentes al disco óptico.

Clasificación de las membranas neovasculares

según su comportamiento angiográfico:

- Predominantemente clásicas. Membranas con patrón en rueda de carro y cuya extensión es mayor del 50\% del tamaño de la lesión.

- Mínimamente clásicas. Membranas con patrón en rueda de carro y cuya extensión es menor del $50 \%$ del tamaño de la lesión.

- Ocultas. No se evidencia patrón típico en rueda de carro. Pueden apreciarse como hiperfluorescencia tardía de origen indeterminado o como desprendimiento fibrovascular del EPR.

Membranas ocultas que han cobrado identidad propia:

- RAP (Retinal Angiomatous Proliferation). Enfermedad neovascular que comienza siendo intrarretiniana, para extenderse al espacio subretiniano y establecer anastomosis retinocoroidea. La angiografía con verde de indocianina es una herramienta útil para su diagnóstico.

- Vasculopatía coroidea polipoidea idiopática. Dilataciones vasculares polipoideas en la coroides que se asocian a cuadros repetidos de sangrado subretiniano. Se evidencian mejor mediante la angiografía con verde de indocianina.

\section{Pruebas especiales}

Angiografía con fluoresceína (AGF)

La clasificación de las membranas neovasculares según su comportamiento angiográfico tuvo una gran importancia en el pasado, porque los resultados tanto con láser térmico como con terapia fotodinámica, eran diferentes según los distintos tipos de membranas. Hoy en día, disponemos de tratamientos farmacológicos (anti-VEGF) que permiten mejorar la agudeza visual en $1 / 3$ de los pacientes con DMAE exudativa, independientemente del tipo 
de membrana. Tampoco es imprescindible la angiografía para valorar la respuesta al tratamiento porque, generalmente, los criterios de retratamiento se basan en la agudeza visual, los hallazgos en la OCT y en la exploración con biomicroscopía del fondo de ojo.

No obstante, es aconsejable realizar una angiografía con fluoresceína, como mínimo, en el momento del diagnóstico, ya que sirve como dato pronóstico: son mucho más agresivas las membranas clásicas que las ocultas. Se podría retrasar el tratamiento de una membrana oculta que no presenta signos de actividad (pérdida de agudeza visual o sangrado) y no se puede retrasar el tratamiento de una membrana clásica, porque el riesgo de progresión y pérdida visual es mucho mayor.

Angiografía con verde de indocianina (AVI)

Este tipo de estudio angiográfico sigue utilizándose en el estudio de las membranas neovasculares ocultas, sobre todo para la identificación de los patrones característicos de la vasculopatía coroidea polipoidea idiopática (VCPI) y la proliferación angiomatosa retiniana (RAP).

\section{Tomografía de coherencia óptica}

Es la prueba por excelencia para el estudio de la DMAE exudativa. Permite un análisis cuantitativo de los signos de actividad y de la respuesta al tratamiento. Además, es una prueba rápida y no invasiva, a diferencia de la angiografía con fluoresceína o con verde de indocianina. Es de gran utilidad no sólo para el diagnóstico de la enfermedad, sino también en el seguimiento posterior, especialmente tras el tratamiento con inyecciones intravítreas de antiangiogénicos. Mediante la OCT podemos detectar incrementos en el espesor de la retina con la presencia de fluido, que pueden preceder a la sintomatología que el paciente note (pérdida de agudeza visual, aumento de metamorfopsia) incluso en semanas.

\section{Diagnóstico diferencial}

Debe establecerse con enfermedades que cursen con un desprendimiento de retina exudativo en el área macular, con sangrado intra o subretiniano o con exudación lipídica profunda. Entre ellas, cabe destacar:

- Coroidopatía central serosa en pacientes con edad superior a 50 años.

- Oclusiones vasculares venosas de la retina antiguas.

- Telangiectasias yuxtafoveales.

- Sangrado subretinano por macroaneurismas o tumores.

\section{Tratamiento: fármacos anti-VEGF}

El tratamiento más eficaz, en este momento, es el tratamiento farmacológico con preparados anti-factor de crecimiento endotelial vascular (VEGF), que es uno de los factores más importantes involucrados en el estímulo angiogénico.

Drogas disponibles:

- Ranibizumab (Lucentis ${ }^{\circledR}$ ). Fracción variable del anticuerpo anti-VEGF.

- Bevacizumab (Avastin ${ }^{\circledR}$ ). Anticuerpo completo anti-VEGF.

- Pegaptanib sódico (Macugen ${ }^{\circledR}$ ). Aptámero contra la isoforma 165 del VEGF.

Estos fármacos se administran por vía intravítrea mediante inyecciones que deben realizarse de forma periódica, ya que su vida media es corta. En este momento, se está trabajando en distintos sistemas de liberación retardada con el objetivo de mantener niveles intravítreos más estables y evitar inyecciones tan frecuentes.

Aunque en los ensayos clínicos que demostraron la eficacia de este tipo de terapia (Lucentis ${ }^{\circledR}$ : ANCHOR y MARINA; Macugen ${ }^{\circledR}$ : VISION) las inyecciones intravítreas se administraron cada mes o cada mes y medio, en este momento la tendencia es intentar espaciar las inyecciones, ya que, en la mayor parte de los casos, éstas deben prolongarse durante años.

\section{ESQUEMA DE TRATAMIENTO DE LA DMAE}

\section{Tratamiento de la DMAE exudativa}

\section{Fotocoagulación láser}

Podremos emplearla en lesiones clásicas bien delimitadas de localización extrafoveal y yuxtafo- 


\section{ALGORITMO 3}

\section{Pautas de tratamiento DMAE exudativa}

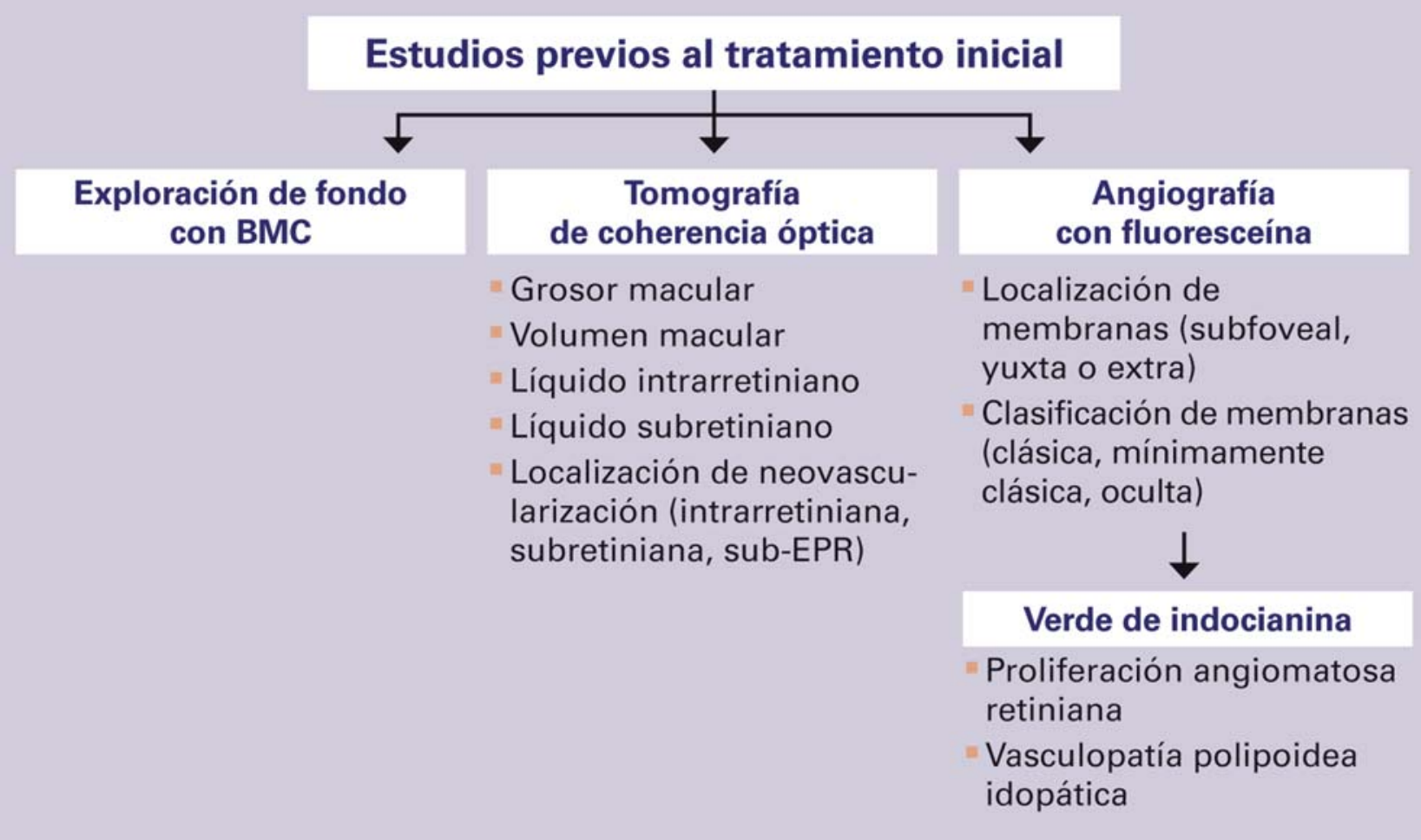

Algoritmos de manejo de la DMAE.

veal. Debemos ser conscientes del alto grado de recidivas (cerca del 50\%) y del riesgo de escotoma irreversible, por lo que, a pesar de que no existen datos científicos del resultado del empleo de inyecciones intravítreas de antiangiogénicos en lesiones yuxtafoveales, muchos autores prefieren el empleo de drogas con capacidad antiangiogénica (1-3). También podríamos considerar esta modalidad de tratamiento en las lesiones peripapilares (4) y en el tratamiento de la VCPI (5) y en los RAPs (6), en los cuales puede usarse con o sin inyección de triamcinolona intravítrea asociada (7).

\section{Cirugía}

La vitrectomía ha ido reduciendo de forma progresiva sus indicaciones en esta enfermedad. En este momento, las más frecuentes son la hemorragia submacular masiva como complicación de la forma exudativa (8), y algunas membranas neovasculares yuxtapapilares cuya extensión no afecte la zona avascular foveal (9).

\section{Terapia fotodinámica}

El empleo de la verteporfina (Visudyne ${ }^{\circledR}$ ) combinado con su estimulación con láser de longitud de onda roja permite mejorar la evolución natural de las lesiones subfoveales con componente clásico mayor del $50 \%$ de la lesión y diámetro total de la lesión inferior a 5.400 micras. La asociación con la inyección intravítrea de triamcinolona permite mejorar los resultados (10-14).

En estas situaciones, el resultado que cabe esperar es una pérdida de visión $(15,16)$, por lo que frente a los resultados obtenidos con nuevas opciones terapéuticas, no debe considerarse hoy en día como tratamiento de primera elección y sólo lo consideraremos ante la imposibilidad de la aplicación del tratamiento intravítreo antiangiogénico. 
ALGORITMO 4

Pautas de tratamiento DMAE exudativa

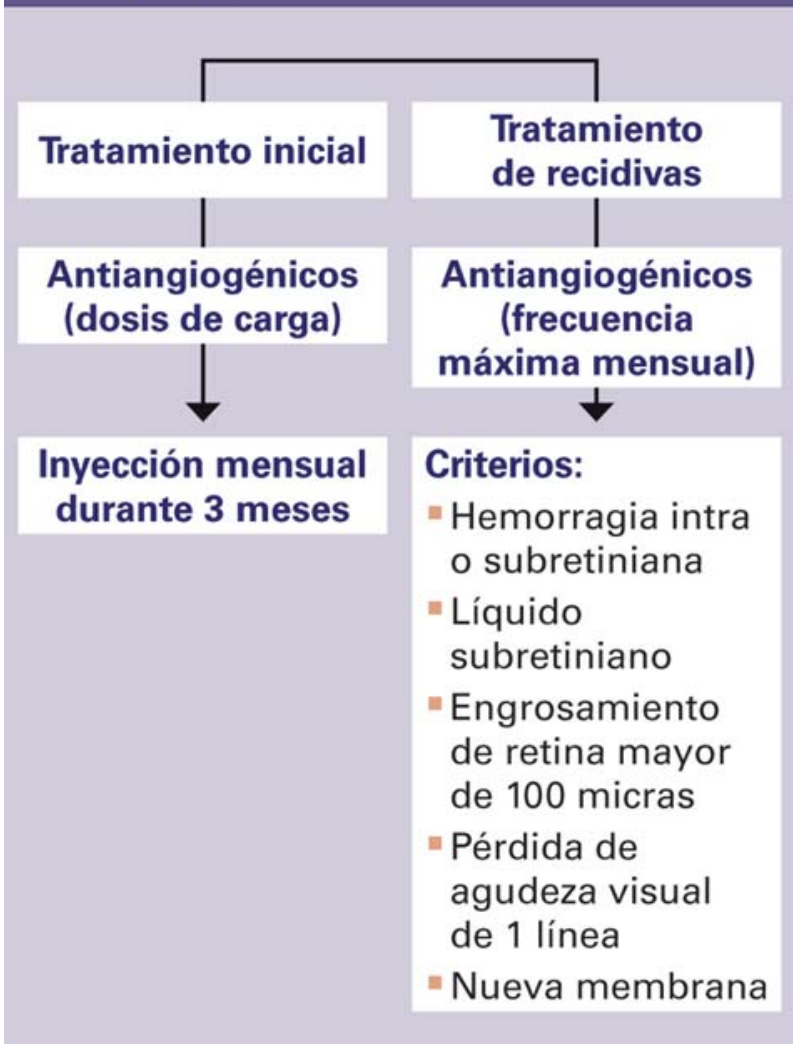

Algoritmos de manejo de la DMAE.

\section{Pegaptanib sódico}

El uso de pegaptanib sódico (Macugen ${ }^{\circledR}$ ) en inyección intravítrea a la dosis de $0,3 \mathrm{mg}$ como tratamiento de lesiones subfoveales activas con un tamaño total inferior a 12 áreas de disco, con componente neovascular mayor del 50\% de la lesión, permite obtener resultados parecidos a la TFD, pero en un abanico más amplio de lesiones neovasculares (17).

La probabilidad de obtener mejorías significativas de visión es bajo (6\%). Por ello, el pegaptanib debería usarse como segunda línea de tratamiento en casos en los que el ranibizumab este contraindicado o el paciente rechace el tratamiento. La ventaja frente a la TFD es que el pegaptanib puede usarse para todo tipo de lesiones, y la terapia fotodinámica en las lesiones ya comentadas $(17,18)$.
La combinación de antiangiogénicos y TFD parece reducir el número de inyecciones intravítreas requeridas, pero los resultados visuales son peores que con el tratamiento antiangiogénico $(19,20)$.

\section{Ranibizumab}

La inyección intravítrea de ranibizumab (Lucentis ${ }^{\circledR}$ ) a la dosis de $0,5 \mathrm{mg}$ permite obtener mejorías significativas de la agudeza visual en lesiones subfoveales, según los datos obtenidos de estudios con máximo nivel de evidencia. Debe ser considerado como el fármaco de primera elección, dada la calificación científica de los resultados que lo avalan (21-28).

La eficacia y la seguridad del ranibizumab fue evaluada por primera vez en el ensayo MARINA de 2 años de duración, que comparaba ranibizumab contra placebo en lesiones ocultas o lesiones mínimamente clásicas. Los pacientes incluidos fueron aleatorizados a recibir una de las dos dosis de ranibizumab, 0,3 mg o 0,5 mg, o simulación de inyección, cada mes durante 24 meses. A los 12 meses, el $95 \%$ de los pacientes que habían recibido ranibizumab perdieron menos de 15 letras ETDRS (3 líneas ETDRS), frente a un $62 \%$ de los pacientes que fueron tratados con simulación de inyección $(\mathrm{P}<0,001)$. Además, el $25 \%$ de los pacientes tratados con $0,3 \mathrm{mg}$ de ranibizumab y el $34 \%$ de los pacientes tratados con $0,5 \mathrm{mg}$ de ranibizumab ganaron 15 letras o más de agudeza visual, comparados con un 5\% de pacientes en el grupo control $(\mathrm{P}<0,001)$. Estas mejorías se mantuvieron a los 2 años. Por primera vez en la historia de los tratamientos de la DMAE exudativa subfoveal, la evolución de la agudeza visual media de los pacientes tratados era hacia la mejoría. Respecto a los efectos adversos, hubo un $1 \%$ de endoftalmitis y un $1,3 \%$ de uveítis.

Posteriormente, el estudio ANCHOR, de 2 años de duración, que comparaba ranibizumab contra TFD en lesiones predominantemente clásicas, mostró resultados muy similares. Los pacientes incluidos fueron aleatorizados a recibir una de las dos dosis de ranibizumab, $0,3 \mathrm{mg}$ o $0,5 \mathrm{mg}$, cada mes o terapia fotodinámica cada 3 meses, según los criterios establecidos para este tratamiento, durante 24 meses. A los 12 meses entre el 94 y el $96 \%$ de los pacientes que recibieron ranibizumab perdieron menos de 15 letras ETDRS (pérdida moderada de 
visión), en comparación con un $64 \%$ de los pacientes que recibieron terapia fotodinámica $(\mathrm{p}<0,001)$. Además, un $36 \%$ de pacientes que recibieron 0,3 $\mathrm{mg}$ de ranibizumab y un $40 \%$ de los pacientes que recibieron $0,5 \mathrm{mg}$ de ranibizumab ganaron 15 letras de ETDRS o más de visión, comparado a solamente un $6 \%$ de los pacientes que recibieron terapia fotodinámica. De la misma forma que en el estudio MARINA, la agudeza visual media de los pacientes tratados también fue hacia una mejoría. Los efectos adversos, como endoftalmitis y uveítis, se mantuvieron por debajo del $1 \%$.

\section{Bevacizumab}

No es un fármaco con indicación aprobada para uso intraocular, ni para el tratamiento de la DMAE húmeda; sin embargo, la experiencia acumulada con miles de pacientes en los últimos años, y los resultados de múltiples series publicadas en revistas con índice de impacto alto, demuestran que el bevacizumab $\left(\right.$ Avastin $\left.{ }^{\circledR}\right)$ puede aportar un beneficio más próximo a los resultados obtenidos con el ranibizumab que a los obtenidos con la terapia fotodinámica y el pegaptanib (29-40).

Nos hallamos ante dos fármacos muy eficaces, uno con aprobación para uso intraocular e indicación para DMAE húmeda y demostrado en ensayos clínicos, y otro sin aprobación intraocular ni indicación para DMAE y demostrado en múltiples series de pacientes. Por esta razón, pensamos que, de acuerdo a la evidencia científica actual, ranibizumab debe ser el fármaco de primera elección frente al bevacizumab.

En aquellos casos que no cumplan los criterios de inclusión para los que fuera aprobado ranibizumab y para los cuales se pueda conseguir el permiso para su uso según la legislación vigente, el bevacizumab puede ser una buena opción.

No puede obviarse tampoco que el coste anual del tratamiento del bevacizumab es muy inferior al del ranibizumab, y ello hace que pueda facilitar, por razones socioeconómicas, el tratamiento a un número mayor de pacientes.

\section{Dosis de «inicio»}

Según los resultados obtenidos en los estudios MARINA y ANCHOR con ranibizumab, el empleo de tres inyecciones consecutivas cada 4 semanas permite obtener el mejor resultado en términos de mejoría de la agudeza visual, aplanándose la curva de evolución de la misma a partir de ese momento (21,22).

Por lo tanto, la recomendación como dosis de «ataque» o de «inicio» es la realización de esta pauta de tres inyecciones intravítreas consecutivas. Sin embargo, debe considerarse la posibilidad de no ser necesario recurrir a las tres dosis, a juicio del especialista, ante situaciones que lo justifiquen.

En el capítulo de tratamiento de la Monografía de la DMAE húmeda, el lector puede consultar con todo tipo de detalles los resultados de los estudios publicados que justifican este algoritmo.

\section{Seguimiento postratamiento}

Tras el tratamiento de la DMAE exudativa con el procedimiento elegido, se plantea cuál debe ser el seguimiento más adecuado, ya que cualquiera de las modalidades terapéuticas empleadas implica un control estricto del paciente con un esquema adecuado al tratamiento empleado. Este hecho viene condicionado porque la enfermedad presenta un elevado número de recidivas con cualquiera de las opciones.

Dependiendo del tratamiento elegido, éste será diferente, pero siempre implicará la realización de todas o algunas de las siguientes pruebas a juicio del médico: una medida de la agudeza visual en idénticas condiciones a lo largo del seguimiento, a ser posible con optotipos ETDRS, biomicroscopía de mácula con lentes de contacto o no contacto, AGF y OCT.

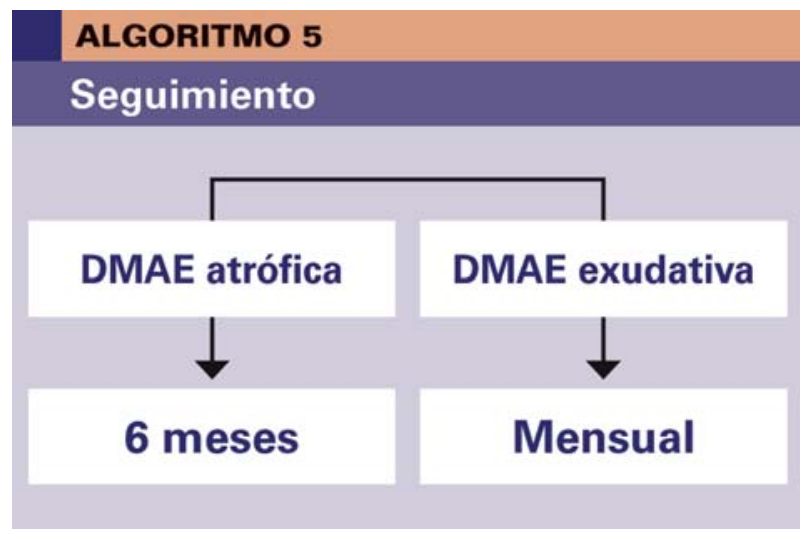

Algoritmos de manejo de la DMAE. 
Tras la realización de una fotocoagulación con láser térmico, a los 15 días deberemos controlar al paciente, incluyendo AGF, que se repetirá entre 4 y 6 semanas según el criterio que el oftalmólogo establezca, dependiendo de los hallazgos de fondo de ojo $(1,2,41)$.

Cuando el procedimiento terapéutico haya sido la TFD con verteporfina, el intervalo recomendado es cada tres meses durante dos años, incluyendo también AGF. Los resultados de los estudios TAP/VIP no permiten conocer si la aplicación de la TFD con intervalo inferior a 10 semanas en los pacientes con enfermedad activa puede proporcionar un beneficio.

Tras la inyección intravítrea de Macugen ${ }^{\circledR}$, el seguimiento se establecerá cada 6 semanas $(17,18)$. Cuando el producto empleado haya sido Lucentis ${ }^{\circledR}$, controlaremos al paciente cada 4 semanas $(21,22)$, al igual que cuando se haya empleado Avastin ${ }^{\circledR}$ $(32,35)$. En estos casos, la información aportada por la OCT permitirá, en muchas ocasiones, evitar la realización de la AGF, a la que podremos recurrir ante casos de duda diagnóstica acerca de si existe o no actividad neovascular.

En cualquier caso, será el criterio del oftalmólogo, según los datos obtenidos en la exploración del paciente, el que decidirá cuál o cuáles de las pruebas son necesarias y cuáles pueden obviarse en cada visita de control.

En el caso de la inyección intravítrea con antiangiogénicos, los máximos niveles de eficacia (estudios MARINA y ANCHOR) fueron conseguidos con inyecciones mensuales durante 2 años de ranibizumab $(21,22)$. Este hecho no es muy factible en la práctica clínica diaria, por lo que se plantea la posibilidad de reducir el número de inyecciones $(42,43)$.

Para ello, se establecieron criterios de retratamiento basados en hallazgos que permitiesen identificar la recidiva o persistencia de actividad de la NVC, ya que los hallazgos publicados con criterios de retratamiento fijo no obtenían buenos resultados visuales (43).

En el estudio PRONTO se empleó: pérdida de 5 letras ETDRS asociado a fluido en la mácula detectado por OCT, un incremento del GCR de al menos 100 micras, aparición de nueva NVC clásica o hemorragia macular o persistencia de fluido detectado por OCT al menos un mes después de la inyección anterior (42).

Este planteamiento implica la realización de una visita de control en el seguimiento del paciente cada cuatro semanas, realizando una nueva inyección ante los mencionados criterios. Según el tipo y agresividad de la lesión, tras 3 controles sin actividad neovascular se podrá buscar el compromiso de alargar los controles de forma individualizada para cada caso. Hasta al menos pasado un año sin enfermedad activa, no deberían alargarse los controles a 3 meses o más. Para ello, deberemos instruir al paciente en la detección, lo más precoz posible, de los síntomas de una recidiva con autocontroles de la visión monocular del ojo afecto. Ante una disminución de la visión o un aumento o aparición de nueva metamorfopsia (la rejilla de Amsler será de gran ayuda para detectar estos cambios por parte del enfermo), deberá acudir lo antes posible a su oftalmólogo.

Cuando se trata del «primer ojo» del paciente, debemos advertir al enfermo de la bilateralidad de la enfermedad, de manera que hay explicarle que en el caso de la forma atrófica tiene tendencia a bilateralidad y simetría, con una velocidad de progresión/crecimiento de 1/10 de diámetro de papila/año. En la forma neovascular, la velocidad de crecimiento es de unos $10 \mu \mathrm{m} /$ día y la tendencia a la bilateralidad, es decir el riesgo del segundo ojo, es de: al $1 .^{\text {er }}$ año: $4 \%$; al 2..$^{\circ}$ año: $10 \%$; al 3. ${ }^{\text {er }}$ año: $17 \%$; y cerca del $25 \%$ a los 5 años.

Ante esta situación, el enfermo debe ser consciente de la importancia del diagnóstico precoz en el segundo ojo y, por lo tanto, acudir a la consulta de forma urgente ante una disminución de visión o cambios en su metamorfopsia. Para ello, la rejilla de Amsler puede ser de utilidad.

\section{BIBLIOGRAFÍA}

1. Macular photocoagulation study group. Argon laser photocoagulation for neovascular maculopathy. Fiveyears results from randomized clinical trials. Arch Ophthalmol 1991; 109: 1109-1114.

2. Macular photocoagulation study group. Argon laser photocoagulation for juxtafoveal choroidal neovascularization. Five-years results from randomized clinical trials. Arch Ophthalmol 1994; 112: 500-509.

3. Macular photocoagulation study group. Laser photocoagulation of subfoveal neovascular lesions in age-related macular degeneration. Results of a randomized clinical trials. Arch Ophthalmol 1991; 109: 1220-1231.

4. Bhatt NS, Al-Shirawi NM, Diamond JG. Krypton laser photocoagulation of peripapillary choroidal neovascular membrane. Ophthalmic Surg Lasers 1999; 30: 56-58.

5. Lai TY, Chan WM, Lam DS. Laser photocoagulation of indocyanine green aniographically identified feeder vessels to idiopathic polypoidal choroidal vasculopathy. Am J Ophthalmol 2004; 138: 693-694. 
6. Johnson TM, Glaser BM. Focal laser ablation of retinal angiomatous proliferation. Retina 2006; 26: 765-772.

7. Roth DB, Scott IU, Gloth JM, Green SN, Yarian DL, Wheatley M. Micropulsed laser photocoagulation and intravitreal triamcinolone acetonide injection for the treatment of retinal angiomatous proliferation. Retina 2007; 27: 1201-1204.

8. Merrill PT, LoRusso FJ, Lomeo MD, Saxe SJ, Khan MM, Lambert HM. Surgical removal of subfoveal choroidal neovascularization in age-related macular degeneration. Ophthalmology 1999; 106: 782-789.

9. Kokame GT, Yamaoka S. Subretinal surgery for peripapillary subretinal neovascular membranes. Retina 2005; 25: 564-569.

10. Treatment of age-related macular degeneration with photodynamic therapy (TAP) Study Group. Photodynamic therapy of subfoveal choroidal neovascularization in agerelated macular degeneration with verteporfin: one-year results of 2 randomized clinical trials - TAP report. Arch Ophthalmol 1999; 117: 1329-1345. Erratum in: Arch Ophthalmol 2000; 118: 488.

11. Verteporfin in Photodynamic Therapy (VIP) Study Group. Photodynamic Therapy of Subfoveal Choroidal Neovascularization in Pathologic Myopia with Verteporfin (oneyear results of a randomized clinical trial - VIP report no. 1. Ophthalmology 2001; 108: 841-852.

12. Bressler NM. Verteporfin therapy of subfoveal choroidal neovascularization in age-related macular degeneration: two-year results of a randomized clinical trial including lesions with occult with no classic choroidal neovascularization-verteporfin in photodynamic therapy report 2. Am J Ophthalmol 2002; 133: 168-169.

13. Ruiz-Moreno JM, Montero J, Barile S, Zarbin M. Photodynamic therapy and high doses intravitreal triamcinolone to treat exudative age-related macular degeneration: One year outcome. Retina 2006; 26: 602-612.

14. Arias L, Garcia-Arumi J, Ramon JM, Badia M, Rubio M, Pujol O. Photodynamic therapy with intravitreal triamcinolone in predominantly classic choroidal neovascularization: one-year results of a randomized study. Ophthalmology 2006; 113: 2243-2250.

15. Kaiser PK, TAP Study Group. Five-year results of verteporfin therapy for subfoveal CNV due to AMD: third year of an open-label extension of the TAP Investigation. Invest Ophthalmol Vis Sci 2003; 44: E-Abstract 1099.

16. Chakravarthy U, Soubrane G, Bandello F, Chong V, Creuzot-Garcher C, Dimitrakos SA, et al. Evolving European guidance on the medical management of neovascular age related macular degeneration. Br J Ophthalmol 2006; 90: 1188-1196.

17. Gragoudas ES, Adamis AP, Cunningham ET Jr, Feinsod M, Guyer DR. Pegaptanib for neovascular age-related macular degeneration. N Engl J Med 2004; 351: $2805-$ 2816.

18. VEGF Inhibition Study in Ocular Neovascularization (V.I.S.I.O.N.) Clinical Trial Group, Chakravarthy U, Adamis AP, Cunningham ET Jr, Goldbaum M, Guyer DR, et al. Year 2 efficacy results of 2 randomized controlled clinical trials of pegaptanib for neovascular age-related macular degeneration. Ophthalmology 2006; 113: 1508-1521.

19. Heier JS, Boyer DS, Ciulla TA, Ferrone PJ, Jumper JM, Gentile RC, et al. Ranibizumab combined with verteporfin photodynamic therapy in neovascular age-related macu- lar degeneration: year 1 results of the FOCUS Study. Arch Ophthalmol 2006; 124: 1532-1542. Erratum in: Arch Ophthalmol 2007; 125: 138.

20. Antoszyk AN, Tuomi L, Chung CY, Singh A; FOCUS Study Group. Ranibizumab combined with verteporfin photodynamic therapy in neovascular age-related macular degeneration (FOCUS): year 2 results. Am J Ophthalmol 2008; 145: 862-874.

21. Rosenfeld PJ, Brown DM, Heier JS, Boyer DS, Kaiser PK, Chung CY, et al. Ranibizumab for neovascular age-related macular degeneration. $N$ Engl J Med 2006; 355: 14191431.

22. Brown DM, Kaiser PK, Michels M, Soubrane G, Heier JS, Kim RY, et al. Ranibizumab versus verteporfin for neovascular age-related macular degeneration. $N$ Engl J Med 2006; 355: 1432-1444.

23. Kaiser PK. Antivascular endothelial growth factor agents and their development: therapeutic implications in ocular diseases. Am J Ophthalmol 2006; 142: 660-668.

24. Kim KJ, Li B, Houck K, Winer J, Ferrara N. The vascular endothelial growth factor proteins: identification of biologically relevant regions by neutralizing monoclonal antibodies. Growth Factors 1992; 7: 53-64.

25. Chen $Y$, Wiesmann $C$, Fuh $G$, Li B, Christinger HW, McKay $P$, et al. Selection and analysis of an optimized anti-VEGF antibody: crystal structure of an affinity-matured Fab in complex with antigen. J Mol Biol 1999; 293: 865-881.

26. Rosenfeld PJ, Schwartz SD, Blumenkranz, MS, Miller JW, Haller JA, Reimann JD, et al. Maximum tolerated dose of a humanized anti-vascular endothelial growth factor antibody fragment for treating neovascular age-related macular degeneration. Ophthalmology 2005; 112: 1048-1053.

27. Michelle Dalton. Lucentis at one year. Eyeworld 2008-5525 11:12:21 http://www.eyeworld.org/printarticle.php?id $=4390$

28. Ip MS, Scott IU, Brown GC, Brown MM, Ho AC, Huang SS, et al. Anti-vascular endothelial growth factor pharmacotherapy for age-related macular degeneration: a report by the American Academy of Ophthalmology. Ophthalmology 2008; 115: 1837-1846.

29. Michels S, Rosenfeld PJ, Puliafito CA, Haraus EN, Venkatraman AS. Systemic bevacizumab (Avastin) therapy for neovascular age-related macular degeneration twelveweek results of an uncontrolled open-label clinical study. Ophthalmology 2005; 112: 1035-1047.

30. Rosenfeld PJ, Fung AE, Puliafito CA. Optical coherence tomography findings after an intravitreal injection of bevacizumab (Avastin) for macular edema from central retinal vein occlusion. Ophthalmic Surg Lasers Imaging 2005; 36: 336-339.

31. Rosenfeld PJ, Moshfeghi AA, Puliafito CA. Optical coherence tomography findings after an intravitreal injection of bevacizumab (Avastin) for neovascular age-related macular degeneration. Ophthalmic Surg Lasers Imaging 2005; 36: 331-335.

32. Spaide RF, Laud K, Fine HF, Klancnik JM Jr, Meyerle CB, Yannuzzi LA, et al. Intravitreal bevacizumab treatment of choroidal neovascularization secondary to age-related macular degeneration. Retina 2006; 26: 383-390.

33. Aisenbrey S, Ziemssen F, Volker M, Gelisken F, Szurman P, Jaissle $G$, et al. Intravitreal bevacizumab (Avastin) for 
occult choroidal neovascularization in age-related macular degeneration. Graefes Arch Clin Exp Ophthalmol 2007; 245: 941-948.

34. Chen E, Kaiser RS, Vander JF. Intravitreal bevacizumab for refractory pigment epithelial detachment with occult choroidal neovascularization in age-related macular degeneration. Retina 2007; 27: 445-450.

35. Rich RM, Rosenfeld PJ, Puliafito CA, Dubovy SR, Davis JL, Flynn HW Jr, et al. Short-term safety and efficacy of intravitreal bevacizumab (Avastin) for neovascular agerelated macular degeneration. Retina 2006; 26: $495-$ 511.

36. Emerson $M V$, Lauer AK, Flaxel CJ, Wilson DJ, Francis PJ, Stout JT, et al. Intravitreal bevacizumab (Avastin) treatment of neovascular age-related macular degeneration. Retina 2007; 27: 439-444.

37. Wu L, Martinez-Castellanos MA, Quiroz-Mercado H, Arevalo JF, Berrocal MH, Farah ME, et al. Twelve-month safety of intravitreal injections of bevacizumab (Avastin $\left.{ }^{\circledR}\right)$ : results of the Pan-American collaborative retina study group (PACORES). Graefes Arch Clin Exp Ophthalmol 2007; 246: 81-87.

38. Cleary CA, Jungkim S, Ravikumar K, Kelliher C, Acheson $R W$, Hickey-Dwyer M. Intravitreal bevacizumab in the tre- atment of neovascular agerelated macular degeneration, 6- and 9-month results. Eye 2008; 22: 82-86.

39. Bashshur ZF, Haddad ZA, Schakal A, Jaafar RF, Saab M, Noureddin BN. Intravitreal bevacizumab for treatment of neovascular age-related macular degeneration: a one-year prospective study. Am J Ophthalmol 2008; 145: 249-256.

40. Chen CY, Wong TY, Heriot WJ. Intravitreal bevacizumab (Avastin) for neovascular age-related macular degeneration: a short-term study. Am J Ophthalmol 2007; 143: 510-512.

41. Macular photocoagulation study group. Laser photocoagulation of subfoveal neovascular lesions of age-related macular degeneration. Update findings from two clinical trials. Ophthalmology 1993; 111: 1200-1209.

42. Fung AE, Lalwani GA, Rosenfeld PJ, Dubovy SR, Michels $S$, Feuer WJ, et al. An optical coherence tomography-guided, variable dosing regimen with intravitreal ranibizumab (Lucentis) for neovascular age-related macular degeneration. Am J Ophthalmol 2007; 143: 566-583.

43. Regillo CD, Brown DM, Abraham P, Yue H, Ianchulev T, Schneider S, et al. Randomized, doublemasked, sham-controlled trial of ranibizumab for neovascular age-related macular degeneration: PIER Study year 1. Am J Ophthalmol 2008; 145: 239-248. 